\title{
Surgical resection significantly promotes the overall survival of patients with hepatocellular carcinoma: a propensity score matching analysis
}

Yaw-Sen Chen ${ }^{4,5 \dagger}{ }^{+}$Pei-Min Hsieh ${ }^{5 \dagger}$, Hung-Yu Lin ${ }^{4,5 \dagger}{ }^{+}$, Chao-Ming Hung ${ }^{4,5}$, Gin-Ho Lo ${ }^{2,4}$, Yao-Chun Hsu ${ }^{2,4}$, I.-Cheng Lu, ${ }^{3,4}$, Chih-Yuan Lee ${ }^{8}$, Tsung-Chin Wu ${ }^{1,4}$, Jen-Hao Yeh 1,2,4, Pojen Hsiao ${ }^{1,4}$, Yu-Chan Li ${ }^{4}$, Ya-Chin Wang ${ }^{1,4}$, Kun-Chou Hsieh ${ }^{5}$ and Chih-Wen Lin ${ }^{1,2,3,4,6,7^{*}}$

\begin{abstract}
Background: The benefits of surgical resection (SR) for various Barcelona Clinic Liver Cancer (BCLC) stages of hepatocellular carcinoma (HCC) remain unclear. We investigated the risk factors of overall survival (OS) and survival benefits of SR over nonsurgical treatments in patients with HCC of various BCLC stages.

Methods: Overall, 2316 HCC patients were included, and their clinicopathological data and OS were recorded. OS was analyzed by the Kaplan-Meier method and Cox regression analysis. Propensity score matching (PSM) analysis was performed.
\end{abstract}

Results: In total, 66 (2.8\%), 865 (37.4\%), 575 (24.8\%) and 870 (35.0\%) patients had BCLC stage 0, A, B, and C disease, respectively. Furthermore, 1302 (56.2\%) of all patients, and 37 (56.9\%), 472 (54.6\%), 313 (54.4\%) and 480 (59.3\%) of patients with BCLC stage $0, A, B$, and C disease, respectively, died. The median follow-up duration time was 20 (range 0-96) months for the total cohort and was subdivided into 52 (8-96), 32 (1-96), 19 (0-84), and 12 (0-79) months for BCLC stages $0, A, B$, and C cohorts, respectively. The risk factors for OS were (1) SR and cirrhosis; (2) SR, cirrhosis, and Child-Pugh (C-P) class; (3) SR, hepatitis B virus (HBV) infection, and C-P class; and (4) SR, HBV infection, and C-P class for the BCLC stage $0, A, B$, and C cohorts, respectively. Compared to non-SR treatment, SR resulted in significantly higher survival rates in all cohorts. The 5 -year OS rates for SR vs. non-SR were $44.0 \%$ versus $28.7 \%, 72.2 \%$ versus $42.6 \%$, $42.6 \%$ versus $36.2,44.6 \%$ versus $23.5 \%$, and $41.4 \%$ versus $15.3 \%$ (all $P$ values $<0.05$ ) in the total and BCLC stage $0, A, B$, and $C$ cohorts, respectively. After PSM, SR resulted in significantly higher survival rates compared to non-SR treatment in various BCLC stages.

Conclusions: SR conferred significant survival benefits to patients with HCC of various BCLC stages and should be considered a recommended treatment for select HCC patients, especially patients with BCLC stage $B$ and C disease.

\footnotetext{
*Correspondence: lincw66@gmail.com

†Yaw-Sen Chen, Pei-Min Hsieh and Hung-Yu Lin have contributed equally

to this work

${ }^{1}$ Division of Gastroenterology and Hepatology, E-Da Dachang Hospital,

I-Shou University, No. 1, Yida Road, Jiaosu Village, Yanchao District,

Kaohsiung 807, Taiwan, ROC

Full list of author information is available at the end of the article
}

(c) The Author(s) 2021. This article is licensed under a Creative Commons Attribution 4.0 International License, which permits use, sharing, adaptation, distribution and reproduction in any medium or format, as long as you give appropriate credit to the original author(s) and the source, provide a link to the Creative Commons licence, and indicate if changes were made. The images or other third party material in this article are included in the article's Creative Commons licence, unless indicated otherwise in a credit line to the material. If material is not included in the article's Creative Commons licence and your intended use is not permitted by statutory regulation or exceeds the permitted use, you will need to obtain permission directly from the copyright holder. To view a copy of this licence, visit http://creativecommons.org/licenses/by/4.0/. The Creative Commons Public Domain Dedication waiver (http://creativecommons.org/publicdomain/zero/1.0/) applies to the data made available in this article, unless otherwise stated in a credit line to the data. 
Keywords: Surgical resection, Hepatocellular carcinoma, Overall survival, Hepatitis B virus, Prognosis

\section{Background}

Hepatocellular carcinoma ( $\mathrm{HCC}$ ) is a major cause of cancer-related death worldwide [1]. HCC staging systems have been developed for treatment and prognostic evaluation [2-7]. The Barcelona Clinic Liver Cancer (BCLC) system is widely utilized because it incorporates tumor burden, liver cirrhosis severity, and patient performance status and is thus advantageous for treatment and prognostic assessment $[4,6,7]$. The BCLC system is approved by the American Association for the Study of Liver Disease (AASLD) and the European Association for the Study of Liver (EASL) $[6,7]$. Patients with stage 0 (very early-stage) and stage A (early-stage) HCC are recommended to undergo surgical resection (SR), while patients with stage B (intermediate-stage) and stage C (advanced-stage) HCC are recommended to undergo transcatheter arterial chemoembolization (TACE) and sorafenib treatment according to the BCLC system. However, the BCLC system is limited because of differences in tumor conditions and heterogeneity in the prognosis of various stages of disease, especially BCLC stages B and C $[8,9]$. Recently, numerous studies, mostly from AsiaPacific countries, have focused on increasing the use of
SR in patients with BCLC stage $B$ and $C$ disease and have demonstrated better overall survival (OS) in patients who have undergone SR as compared to patients with nonsurgical treatments [9-13]. However, some studies have shown that TACE is not inferior to SR for patients with operable BCLC stage B and C HCC [14]. The advantages of SR over nonsurgical treatments for $\mathrm{HCC}$ of various BCLC stages are still unknown. Furthermore, several prognostic factors, including age, treatment, liver function, tumor size, and etiology, are associated with OS in HCC patients $[15,16]$. However, the prognostic factors for survival in HCC patients remain elusive. This study aimed to investigate the risk factors of OS and the potential benefits of SR over nonsurgical treatments in a large cohort of HCC patients.

\section{Methods}

Patients and follow-up

We retrospectively collected information on 2759 patients diagnosed with HCC between 2010 and 2016 at E-Da Hospital, I-Shou University, Kaohsiung, Taiwan and 543 patients were excluded (Fig. 1). The study was conducted in accordance with the guidelines of the

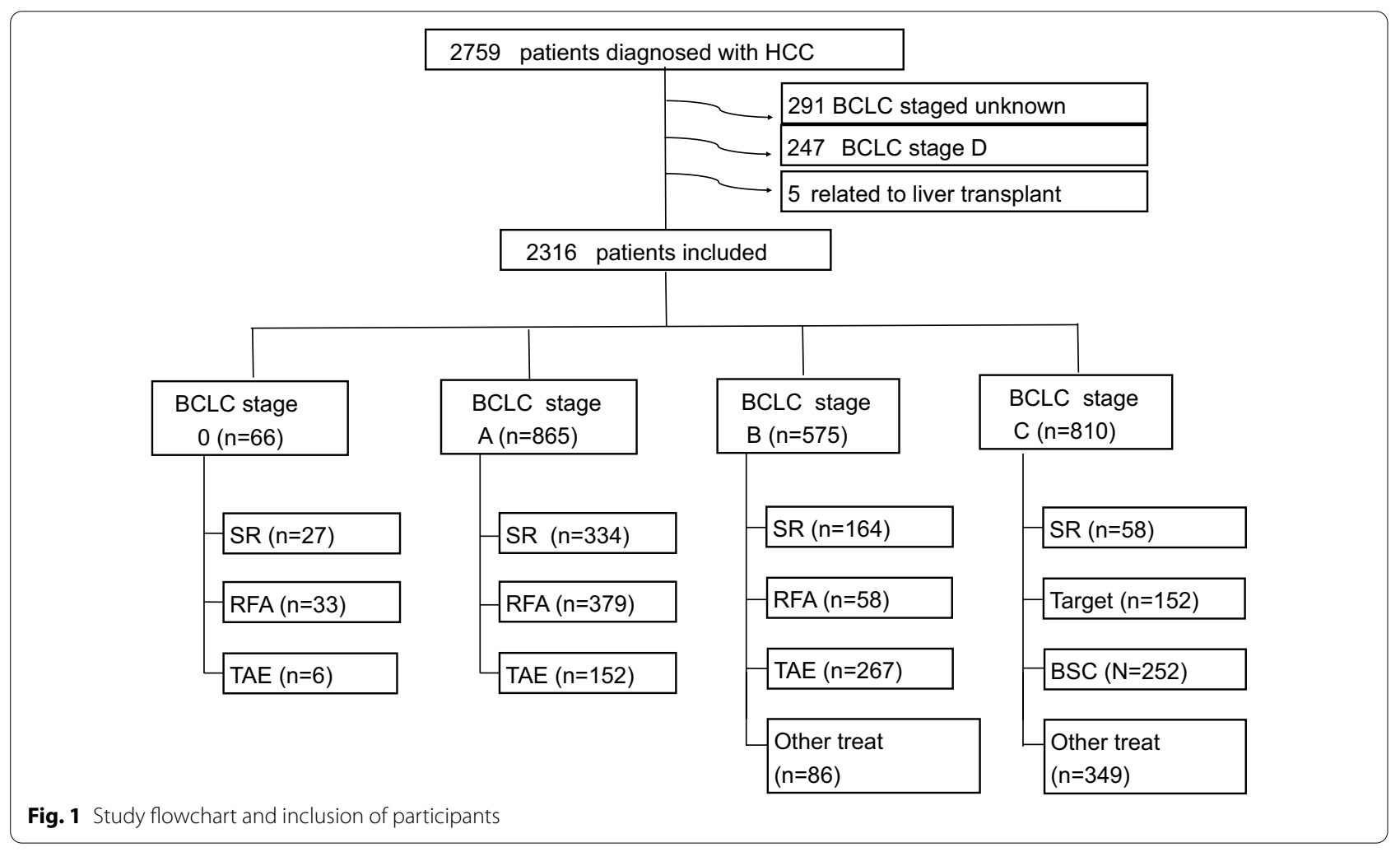


International Conference on Harmonization for Good Clinical Practice and was approved by the Ethics Committee of E-Da Hospital, I-Shou University (EMRP107-130). Patients were diagnosed with HCC based on histological confirmation or at least one typical imaging method according to the recommendations of the AASLD [6]. OS was defined as the time from the date of diagnosis to the date of death or last follow-up, and the last follow-up was in December 2017. SR was defined as hepatic resection for HCC. Non-SR treatments included radiofrequency ablation (RFA), TACE, hepatic artery infusion chemotherapy (HAIC), targeted therapy (sorafenib), radiotherapy (RT) and best supportive care (BSC). Patients with older age received RFA or TACE in BCLC stage 0 and A because patients refused SR due to the possibility of older age or high risk for post-operative morbidity and mortality. Patients underwent SR in BCLC stage $B$ because of patients with resectable $\mathrm{HCC}$ lesions and indocyanine green is less than $10 \%$. Patients underwent SR in BCLC stage $C$ because patients were resectable HCC lesions and indocyanine green is less than 10\%. Clinicographic data, smoking, excessive alcohol use, hepatitis status, liver cirrhosis, Child-Pugh (C-P) class, tumor size, tumor number, and vascular invasion, were examined. Tumor number, tumor size and vascular invasion were mostly determined based on radiologic findings and confirmed by pathologic findings if appropriate. Liver cirrhosis was diagnosed based on pathologic findings and/or evaluated by ultrasound, computed tomography, or magnetic resonance imaging. The functional status of the liver was evaluated using the $\mathrm{C}-\mathrm{P}$ scoring system[17].

\section{Data analysis and statistics}

All statistical analyses were performed using SPSS ver. 18.0 (SPSS, Chicago, IL, USA). Numerical data were expressed as medians and ranges. Categorical data were described using numbers and percentages. OS was determined using the Kaplan-Meier method and compared among patients with HCC of various BCLC stages and among patients receiving different treatments. Cox proportional hazards regression analysis of OS in HCC patients was performed according to BCLC stages. Variables including sex, age, smoking, alcohol use, HBV infection, hepatitis $\mathrm{C}$ virus (HCV) infection, cirrhosis, $\mathrm{C}-\mathrm{P}$ class, tumor size, tumor number, and treatment were incorporated into the Cox regression analysis. Moreover, we used logistic regression to generate propensity score matching (PSM) with with sex, age, cirrhosis, C-P class, tumor size, and tumor number for all patients of various BCLC stage in order to reduce bias in our analyses. The two treatment groups were matched with the control group according to the generated PSM using a caliper width of 0.02 . On the completion of matching, the baseline covariates were compared using the paired $t$-test or Mann-Whitney $U$ test for continuous variables and the chi-square test for categorical variables. A $P$ value $<0.05$ was used to determine statistical significance.

\section{Results}

\section{Baseline demographic data}

A total of 2316 HCC patients were included in this study (Fig. 1). The demographic and clinicopathological features of the 2316 patients $(75.5 \%$ male, median age of 63 years) are shown in Table 1. Regarding the etiology of $\mathrm{HCC}, 71.6 \%$ of the patients had HBV infection, $30.6 \%$ had HCV infection, and $37.5 \%$ had excessive alcohol use. Approximately $37.9 \%$ of patients had liver cirrhosis, and of those patients, $60.8 \%$ had $\mathrm{C}-\mathrm{P}$ class A disease. The mean tumor size was $6.1 \mathrm{~cm}$ and the mean tumor number was 2.1 tumors. Moreover, 1302 patients (56.2\%) were mortality and the median follow-up time was 22 (range, 1-96) months.

\section{Overall survival of patients in the total and various BCLC stage cohorts}

Of the 2316 patients, 1302 (56.2\%) died, and the median follow-up duration was 20 (range, 1-96) months (Table 1 ). The mortality rate was $35.5 \%$ per person-year. The cumulative OS rates at 5 years were $32.5 \%$ (Fig. 2a). SR was performed in 538 (23.2\%) patients, and the OS was significantly better in these patients than in non-SR patients. The cumulative OS rates at 5 years in the SR and non-SR groups were 44.0 and $28.7 \%$, respectively $(P<0.001$, Fig. 2b). Survival was significantly higher in the BCLC stage 0 cohort than in the BCLC stage A, $\mathrm{B}$, and $\mathrm{C}$ cohorts $(P<0.05)$. The cumulative $\mathrm{OS}$ rates at 5 years in the BCLC stage $0, \mathrm{~A}, \mathrm{~B}$, and $\mathrm{C}$ cohorts were $59.5 \%, 38.7 \%, 31.6$ and $23.4 \%$, respectively (Fig. 2c). For patients receiving SR, survival was significantly higher in the BCLC stage 0 cohort than in the BCLC stage A, $\mathrm{B}$, and $\mathrm{C}$ cohorts $(P<0.01)$. The cumulative OS rates for SR patients at 5 years in the BCLC stage $0, \mathrm{~A}, \mathrm{~B}$, and $\mathrm{C}$ cohorts were $72.2 \%, 42.6 \%, 44.6$ and $41.4 \%$, respectively (Fig. 2d).

\section{Overall survival of patients with BCLC stage 0 disease}

Among the $66 \mathrm{HCC}$ patients with BCLC stage 0 disease, 37 (56.9\%) died, and the median follow-up duration was 52 (range, 8-96) months (Table 1). For the multivariate analysis, Cox proportional hazards modeling showed that patients with cirrhosis were associated with lower survival compared to patients without cirrhosis (hazard ratio $[\mathrm{HR}]: 0.60$; $95 \%$ confidence interval $[\mathrm{CI}]$ : 0.42-0.72, $P=0.006$ ), while patients undergoing SR were associated with higher survival compared to patients without SR 

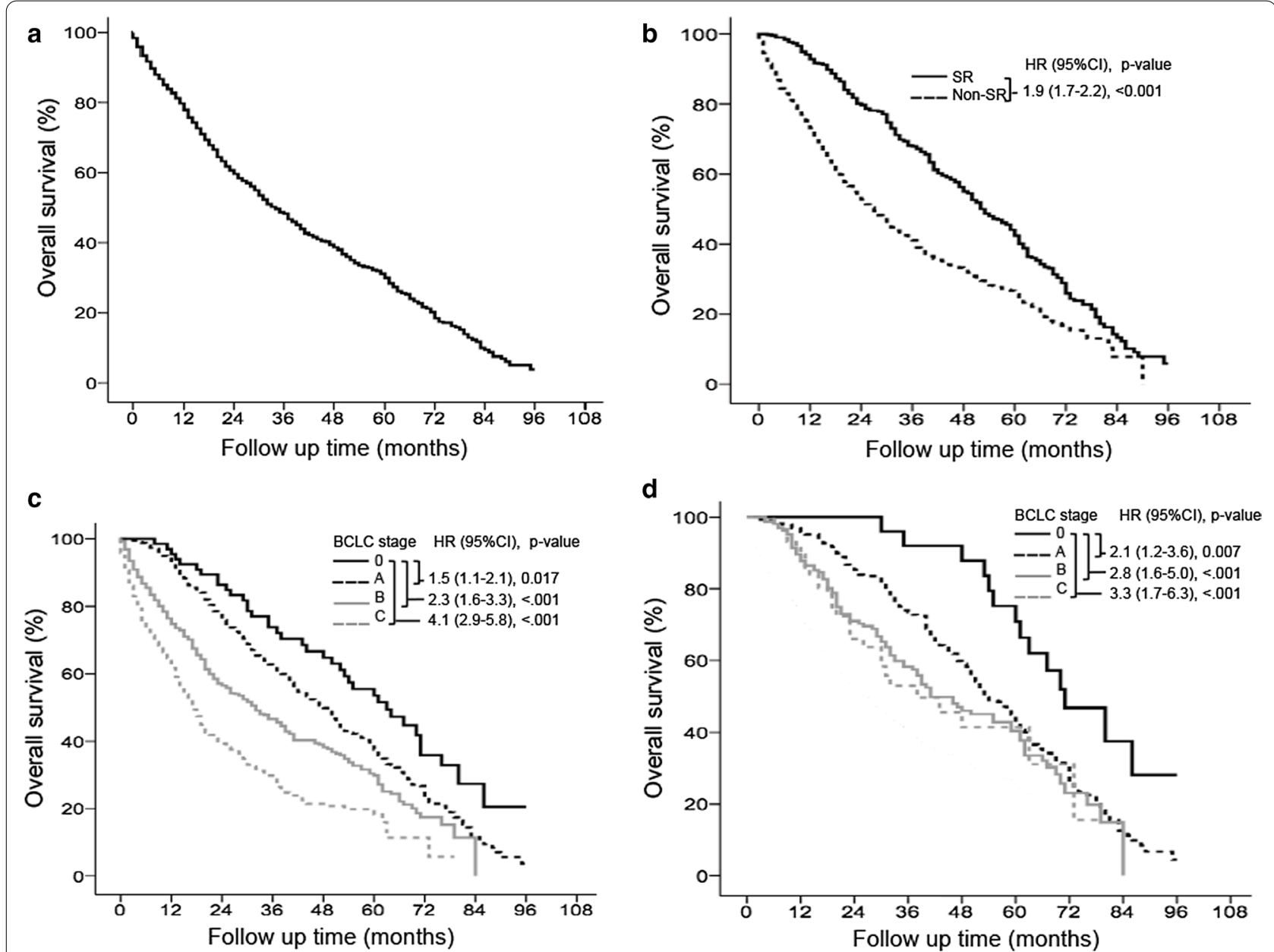

Fig. 2 Overall survival in patients with $B C L C$ stage $B$ and $C$ disease. Surgical resection (SR) resulted in significantly higher overall survival (OS) rates than non-SR treatments in BCLC stage $B(P<0.05)(\mathbf{a})$. After propensity score matching (PSM), SR still had significantly higher OS rates than non-SR treatments in BCLC stage $B(P<0.05)(\mathbf{b})$. SR resulted in significantly higher OS rates than non-SR treatments in BCLC stage $C(P<0.05)(\mathbf{c})$. After PSM, SR still had significantly higher OS rates than non-SR treatments in BCLC stage $C(P<0.05)(\mathbf{d})$

(HR: 2.31; 95\% CI: $1.22-4.61, P=0.019)$ (Table 2). There are significant differences in age and HBV infection between SR and non-SR treatments in Table 3. Twentyseven (40.9\%) patients underwent SR and $39(59.1 \%)$ received non-SR treatments. Patients undergoing SR had significantly higher survival rates than patients receiving nonsurgical treatment $(P=0.019)$, RFA (HR: 2.1; 95\% CI: $1.1-4.4, P=0.037$ ), or TACE (HR: 4.3 ; $95 \%$ CI: 1.3-13, $P=0.015$ ) (Fig. 3a and Additional file 1: Fig. S1A). The cumulative OS rates at 5 years in the SR and non-SR treatments were 72.2 and $42.6 \%$, respectively (Fig. 3a). The basic demographic data of patients with HCC of various BCLC stages between SR and non-SR treatments after PSM was not significant difference and was shown in Table 4. After PSM, patients undergoing SR had significantly higher survival rates than patients receiving nonsurgical treatment $(P=0.01)$. The cumulative OS rates at
5 years in the SR and non-SR treatments were 72.2 and $31.4 \%$, respectively (Fig. 3b).

\section{Overall survival of patients with $B C L C$ stage $A$ disease}

Among the $865 \mathrm{HCC}$ patients with BCLC stage A disease, $472(54.6 \%)$ died, and the median follow-up duration was 32 (range, 1-96) months (Table 1). For the multivariate analysis, patients with cirrhosis and $\mathrm{C}-\mathrm{P}$ class $\mathrm{B}$ were associated with lower survival compared to patients without cirrhosis (HR: 0.55; 95\% CI: 0.39-0.78, $P=0.001$ ) and patients with $\mathrm{C}-\mathrm{P}$ class A (HR: 0.55; 95\% CI: $0.43-0.71, P<0.001)$, respectively. Additionally, patients undergoing SR were associated with higher survival compared to patients without SR (HR: 1.41; 95\% CI: 1.17-1.70, $P<0.0001$ ) (Table 2). There are significant differences in sex, age, smoking, and tumor number 
Table 1 Basic demographic data of patients with hepatocellular carcinoma of different BCLC stages

\begin{tabular}{|c|c|c|c|c|c|}
\hline Variable & Total $(\mathrm{N}=2316)$ & $\begin{array}{l}\text { BCLC stage } 0 \\
(\mathrm{~N}=66)\end{array}$ & $\begin{array}{l}\text { BCLC stage } A \\
(\mathrm{~N}=865)\end{array}$ & $\begin{array}{l}\text { BCLC stage } B \\
(\mathrm{~N}=575)\end{array}$ & $\begin{array}{l}\text { BCLC stage } C \\
(\mathrm{~N}=810)\end{array}$ \\
\hline Male & $1748(75.5)$ & $46(69.7)$ & 605 (69.6) & $437(76.0)$ & $660(81.5)$ \\
\hline Age (years) & 63 (19-99) & $62(23-85)$ & $63(28-92)$ & $64(30-91)$ & $61(19-99)$ \\
\hline Smoking & $949(41.0)$ & $28(42.4)$ & $316(36.5)$ & $232(40.3)$ & $373(46.0)$ \\
\hline Alcohol use & $868(37.5)$ & $22(33.3)$ & $283(32.7)$ & 218 (37.9) & $345(42.6)$ \\
\hline HBV positive & $1658(71.6)$ & $44(66.7)$ & $599(69.2)$ & $414(72.0)$ & $601(74.2)$ \\
\hline HCV positive & $696(30.1)$ & $14(21.2)$ & $253(29.2)$ & $172(29.9)$ & $257(31.7)$ \\
\hline Cirrhosis & $1395(60.2)$ & $43(65.2)$ & $511(59.1)$ & $334(58.1)$ & $507(62.6)$ \\
\hline Child-Pugh class A & $877(37.9)$ & $66(100)$ & $277(32.0)$ & $225(39.1)$ & $309(38.1)$ \\
\hline Tumor size (cm) & $6.1(1.0-27)$ & $1.7(1.0-2.0)$ & $2.9(1.0-5.0)$ & $7.5(1.2-21)$ & $9.4(1.2-27)$ \\
\hline Tumor number & $2.1(1-11)$ & $1(1-1)$ & $1.5(1-3)$ & $2.5(1-11)$ & $2.7(1-11)$ \\
\hline $\begin{array}{l}\text { Treatment, } \\
\text { surgery }\end{array}$ & $538(23.2)$ & $27(40.9)$ & $289(33.4)$ & $164(28.5)$ & $58(7.2)$ \\
\hline Mortality & $1302(56.2)$ & $37(56.9)$ & $472(54.6)$ & $313(54.4)$ & $480(59.3)$ \\
\hline $\begin{array}{l}\text { Median follow-up } \\
\text { duration (months) }\end{array}$ & $20(0-96)$ & $52(8-96)$ & $32(1-96)$ & $19(0-84)$ & $12(0-79)$ \\
\hline
\end{tabular}

BCLC stage: Barcelona clinic liver cancer; HBV: Hepatitis B virus; HCV: Hepatitis C virus; OS: Overall survival; Number (\%); Mean (range)

between SR and non-SR treatments in Table 3. Besides, 334 (38.6\%) patients underwent SR, 531 (61.4\%) received non-SR treatments. Patients undergoing SR had significantly higher survival rates than patients receiving nonSR treatments $(P<0.0001)$, RFA (HR: 1.2 ; 95\% CI: $1.1-1.5$, $P=0.041$ ), or TACE (HR: $1.7 ; 95 \%$ CI: $1.3-2.2, P<0.001$ ) (Fig. 3c and Additional file 1: Fig. S1B). The cumulative OS rates at 5 years in the SR and non-SR treatments were 42.6 and $36.2 \%$, respectively (Fig. 3c). After PSM, patients undergoing SR had significantly higher survival rates than patients receiving non-SR treatments $(P=0.04)$. The cumulative OS rates at 5 years in the SR and non-SR treatments were 42.6 and $41.8 \%$, respectively (Fig. $3 \mathrm{~d}$ ).

\section{Overall survival of patients with BCLC stage $B$ disease}

Among the $575 \mathrm{HCC}$ patients with BCLC stage B disease, 313 (54.4\%) died, and the median follow-up duration was 19 (range, 1-84) months (Table 1). For the multivariate analysis, patients with $\mathrm{HBV}$ infection and $\mathrm{C}-\mathrm{P}$ class $\mathrm{B}$ were associated with lower survival compared to patients without HBV infection (HR: 0.43; 95\% CI: 0.27-0.71, $P=0.001$ ) and patients with C-P class A (HR: 0.41; 95\% CI: 0.28-0.61, $P<0.001)$, respectively. Patients undergoing SR were associated with higher survival compared to patients without SR (HR: 2.10; 95\% CI: 1.56-2.82, $P<0.001$ ) (Table 2). There are significant differences in sex and tumor size between SR and non-SR treatments in Table 3. In addition, 164 (28.5\%) patients underwent SR and 411 (71.5\%) received non-SR treatments. Patients undergoing SR had significantly higher survival rates than patients receiving non-SR treatments $(P<0.0001)$, RFA (HR: $1.4 ; 95 \%$ CI: $1.1-2.2, P=0.043)$, TACE (HR:
1.7; 95\% CI: $1.3-2.2, P<0.001$ ), or other treatments (HR: 2.3; 95\% CI: $1.7-3.3, P<0.001$ ) (Fig. $4 \mathrm{a}$ and Additional file 1: Fig. S1C). The cumulative OS rates at 5 years in the SR and non-SR treatments were 40.4 and $23.5 \%$, respectively (Fig. 4a). After PSM, patients undergoing SR had significantly higher survival rates than patients receiving non-SR treatments $(P<0.001)$. The cumulative OS rates at 5 years in the SR and non-SR treatments were 40.4 and $18.2 \%$, respectively (Fig. $4 \mathrm{~b}$ ).

\section{Overall survival of patients with $B C L C$ stage $C$ disease}

Among the $810 \mathrm{HCC}$ patients with BCLC stage C disease, $313(54.4 \%)$ died, and the median follow-up duration was 12 (range, 1-79) months (Table 1). For the multivariate analysis, patients with $\mathrm{HBV}$ infection and $\mathrm{C}-\mathrm{P}$ class $\mathrm{B}$ were associated with lower survival compared to patients without HBV infection (HR: 0.40; 95\% CI: $0.27-0.58, P<0.001)$ and patients with $\mathrm{C}-\mathrm{P}$ class A (HR: 0.40; 95\% CI: 0.0.29-0.55, $P<0.001$ ), respectively. Patients undergoing SR were associated with higher survival compared to patients without SR (HR: 3.10; 95\% CI: 2.02-4.70, $P<0.001$ ) (Table 2). There are significant differences in alcohol use and cirrhosis between SR and non-SR treatments in Table 3. Fifty-eight (7.2\%) patients underwent SR and 752 (92.8\%) patients received nonSR treatments. Patients undergoing SR had significantly higher survival rates than patients receiving non-SR treatments, RFA, TACE, target therapy, RT, HAIC, and other treatments (all $P<0.05$ ) (Fig. 4c and Additional file 1: Fig. S1D). The cumulative OS rates at 5 years in the SR and non-SR treatments were 41.4 and $15.3 \%$, respectively (Fig. 4c). After PSM, patients undergoing SR had 
Table 2 Cox regression analysis of overall survival in patients with hepatocellular carcinoma of different BCLC stages

\begin{tabular}{|c|c|c|c|c|c|c|c|c|}
\hline \multirow[t]{2}{*}{ Variable } & \multicolumn{2}{|c|}{ BCLC stage $0(\mathrm{~N}=66)$} & \multicolumn{2}{|c|}{ BCLC stage $A(N=865)$} & \multicolumn{2}{|c|}{$\mathrm{BCLC}$ stage $B(\mathrm{~N}=575)$} & \multicolumn{2}{|c|}{ BCLC stage $C(N=810)$} \\
\hline & $\mathrm{HR}(95 \% \mathrm{Cl})$ & $P$ value & HR (95\% Cl) & $P$ value & $\mathrm{HR}(95 \% \mathrm{Cl})$ & $P$ value & $\mathrm{HR}(95 \% \mathrm{Cl})$ & $P$ value \\
\hline \multicolumn{9}{|l|}{ Sex } \\
\hline Female & 1 & & 1 & & 1 & & 1 & \\
\hline Male & $0.70(0.85-1.98)$ & 0.79 & $0.81(0.64-1.03)$ & 0.09 & $0.95(0.68-1.31)$ & 0.76 & $0.90(0.67-1.21)$ & 0.49 \\
\hline \multicolumn{9}{|l|}{ Age (years) } \\
\hline$<60$ & 1 & & 1 & & 1 & & 1 & \\
\hline$\geq 60$ & $0.83(0.61-1.11)$ & 0.22 & $0.84(0.64-1.09)$ & 0.19 & $0.71(0.50-1.01)$ & 0.06 & $0.81(0.59-1.09)$ & 0.17 \\
\hline \multicolumn{9}{|l|}{ Smoking } \\
\hline Yes & 1 & & 1 & & 1 & & 1 & \\
\hline No & $0.52(0.22-1.23)$ & 0.14 & $0.59(0.08-4.41)$ & 0.61 & $0.89(0.37-2.20)$ & 0.82 & $0.81(0.30-2.19)$ & 0.68 \\
\hline \multicolumn{9}{|l|}{ Alcohol use } \\
\hline Yes & 1 & & 1 & & 1 & & 1 & \\
\hline No & $0.70(0.10-6.25)$ & 0.67 & $0.75(0.10-5.59)$ & 0.78 & $0.82(0.55-1.22)$ & 0.33 & $0.52(0.19-1.42)$ & 0.20 \\
\hline \multicolumn{9}{|l|}{ HBV } \\
\hline Negative & 1 & & 1 & & 1 & & 1 & \\
\hline Positive & $0.30(0.04-1.21)$ & 0.25 & $0.22(0.03-1.62)$ & 0.14 & $0.43(0.27-0.71)$ & 0.001 & $0.40(0.27-0.58)$ & $<0.001$ \\
\hline \multicolumn{9}{|l|}{$\mathrm{HCV}$} \\
\hline Negative & 1 & & 1 & & & & 1 & \\
\hline Positive & $0.95(0.73-1.10)$ & 0.49 & $0.51(0.07-3.78)$ & 0.51 & $0.88(0.66-1.18)$ & 0.41 & $0.89(0.41-1.12)$ & 0.35 \\
\hline \multicolumn{9}{|l|}{ Cirrhosis } \\
\hline Absent & 1 & & 1 & & 1 & & 1 & \\
\hline Present & $0.60(0.42-0.72)$ & 0.006 & $0.55(0.39-0.78)$ & 0.001 & $0.91(0.59-1.40)$ & 0.66 & $0.87(0.53-1.43)$ & 0.58 \\
\hline \multicolumn{9}{|l|}{ Child-Pugh class } \\
\hline A & & & 1 & & 1 & & 1 & \\
\hline B & & & $0.55(0.43-0.71)$ & $<0.001$ & $0.41(0.28-0.61)$ & $<0.001$ & $0.40(0.29-0.55)$ & $<0.001$ \\
\hline Tumor size & $0.98(0.97-1.01)$ & 0.238 & $1.06(0.94-1.20)$ & 0.289 & $1.03(0.92-1.15)$ & 0.609 & $0.98(0.96-1.01)$ & 0.238 \\
\hline Tumor number & $0.95(0.96-1.02)$ & 0.058 & $1.01(0.83-1.20)$ & 0.971 & $1.06(0.91-1.24)$ & 0.391 & $0.94(0.94-1.05)$ & 0.052 \\
\hline \multicolumn{9}{|l|}{ Treatment } \\
\hline Non-surgery & 1 & & 1 & & 1 & & 1 & \\
\hline Surgery & $2.31(1.22-4.61)$ & 0.019 & $1.41(1.17-1.70)$ & $<0.001$ & $2.10(1.56-2.82)$ & $<0.001$ & $3.10(2.02-4.70)$ & $<0.001$ \\
\hline
\end{tabular}

BCLC stage: Barcelona clinic liver cancer; HR: Hazard ratio; Cl: Conference incidence; HBV: Hepatitis B virus; HCV: Hepatitis C virus

significantly higher survival rates than patients receiving non-SR treatments $(P<0.001)$. The cumulative OS rates at 5 years in the SR and non-SR treatment groups were 41.4 and $1.8 \%$, respectively (Fig. $4 d$ ).

\section{Discussion}

In this large cohort study, we analyzed 2316 HCC patients to identify the prognostic factors and treatments affecting OS. Our results demonstrated that the risk factors for OS were SR, cirrhosis, C-P class, and HBV infection within groups with different BCLC stages (Table 2). SR resulted in significantly higher OS rates than non-SR treatments among patients with $\mathrm{HCC}$ of various BCLC stages. After PSM, SR still had significantly higher OS rates than non-SR treatments among patients with HCC of various BCLC stages. As SR conferred significant survival benefits to patients with $\mathrm{HCC}$ of different BCLC stages, it should be considered a recommended treatment for selected HCC patients, especially patients with BCLC stage B and C disease.

SR, RFA and liver transplantation are the recommended treatment modalities for very early- and early-stage HCC $[6,7]$. Several studies have shown that SR results in better long-term OS than RFA in very early- and early-stage HCC [18-20]. Consistent with previous studies [18-20], our results demonstrated that SR resulted in significantly higher OS rates when compared to non-SR treatments especial in RFA treatment in patients with BCLC stage 0 and $\mathrm{A}$ disease.

TACE is recommended as a standard of care for the treatment of patients with BCLC stage B disease [6, 7]. Several HCC experts have proposed four substages based on the Eastern Cooperative Oncology Group (ECOG) performance, C-P class, and "up-to-7" criteria within 


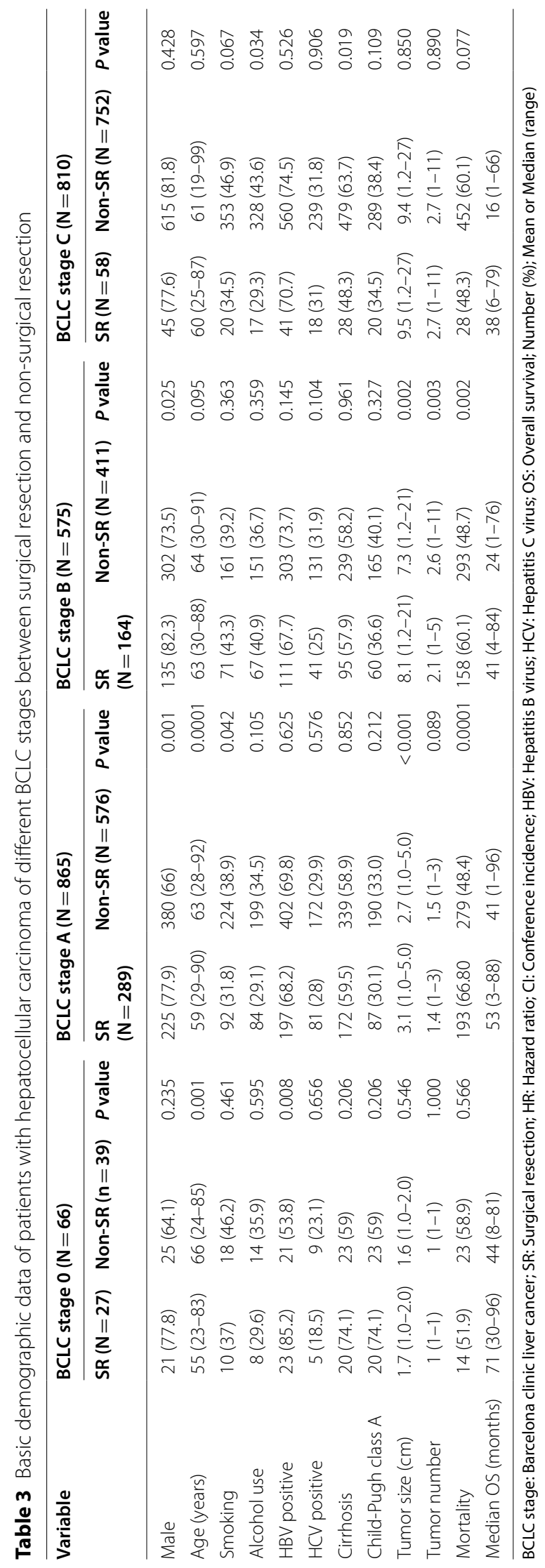




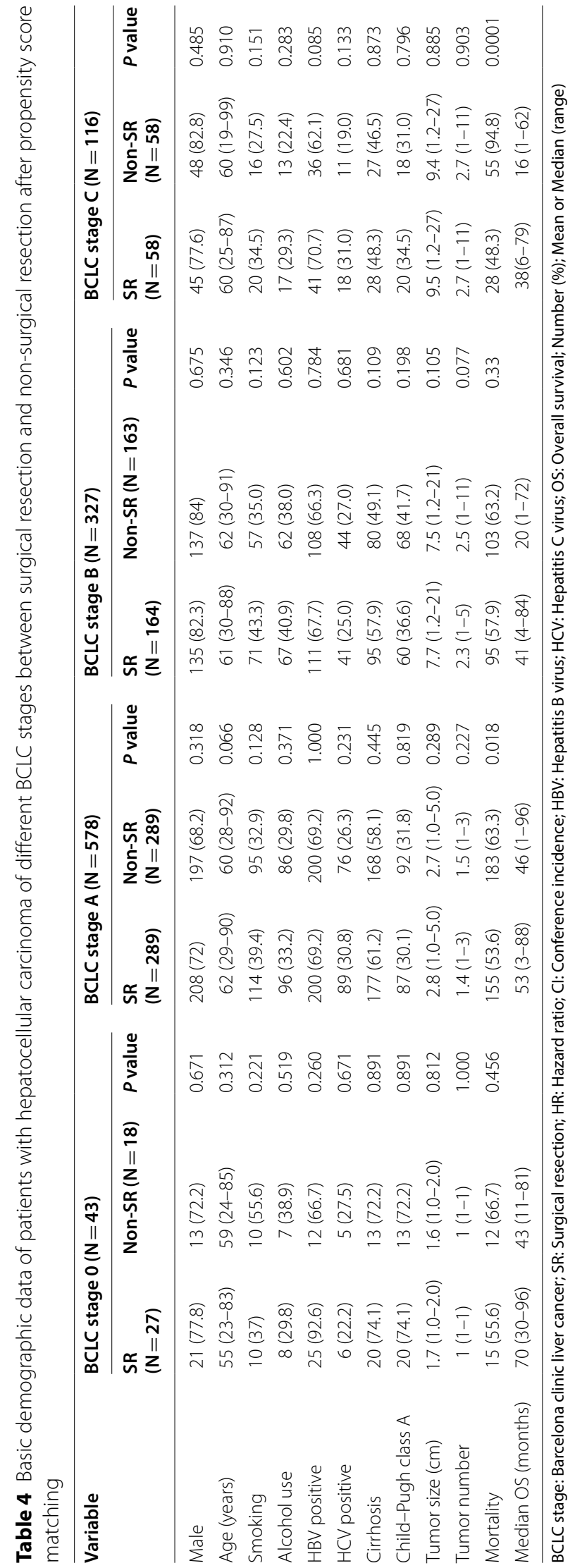



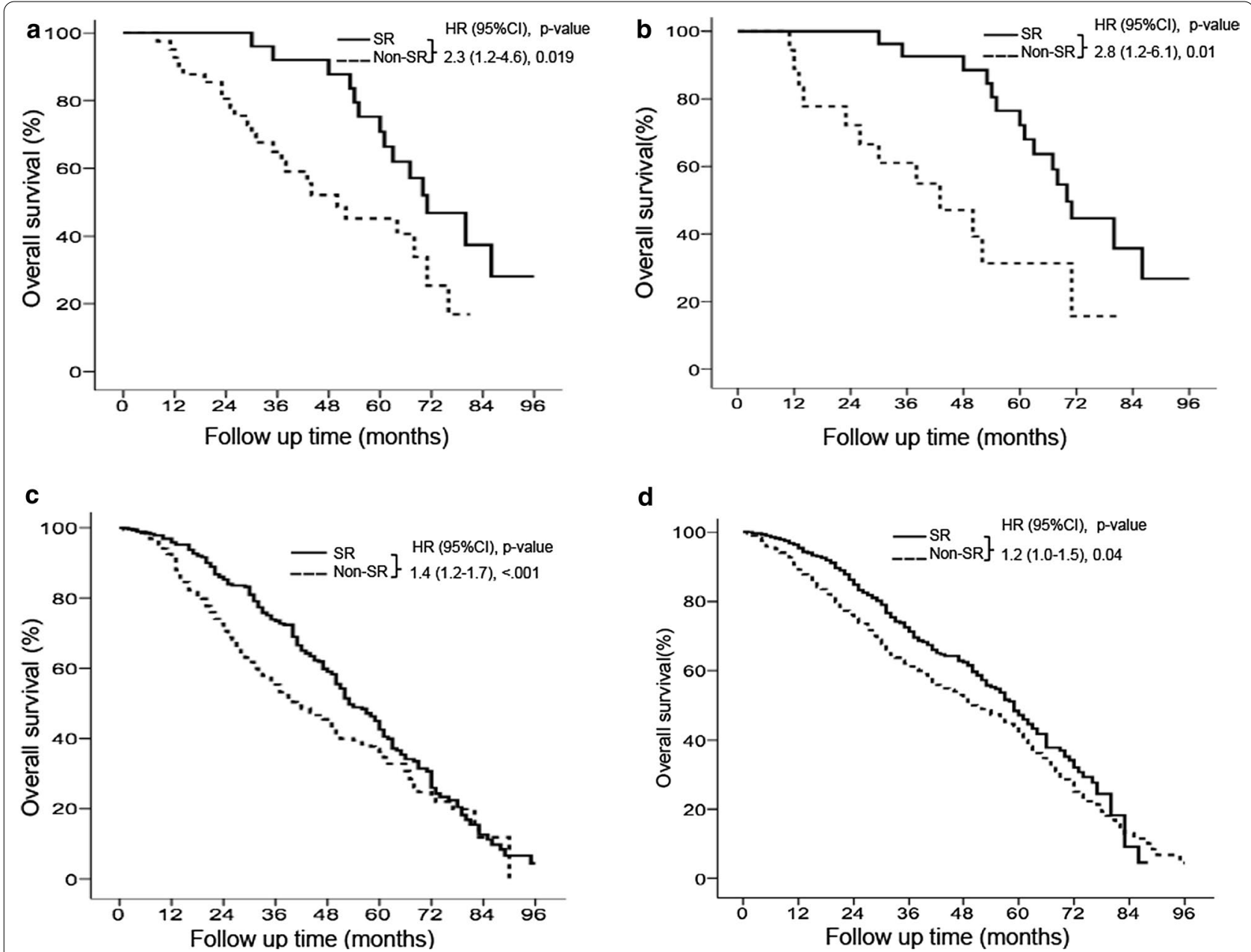

Fig. 3 Overall survival of total cohort in various BCLC stages. Overall survival (OS) of total cohort $(n=2316)(\mathbf{a})$. Surgical resection (SR) resulted in significantly higher OS rates than non-SR treatments in all $2316 \mathrm{HCC}$ patients $(P<0.05)(\mathbf{b})$. OS rates based on Cox regression analysis in patients with HCC of various BCLC stages. Patients with stage 0 disease had significantly better OS than patients stage $A$, $B$, and $C$ disease (c). OS rates based on Cox regression analysis in patients with HCC of various BCLC stages undergoing SR. SR resulted in significantly better OS in patients with stage 0 than in patients with stage A, B, and C disease (d)

BCLC stage B disease [21]. However, these criteria mostly indicate benefits from TACE. Based on the great improvements in surgical techniques and perioperative care, some treatments may not be suitable for patients with intermediate- and advanced-BCLC stage HCC. Our results showed that $S R$ resulted in a significantly higher OS rate than non-SR Treatments including RFA, TACE, and other treatments in patients with BCLC stage B disease. Similarly, several studies from both Western and Eastern countries have demonstrated that SR results in higher long-term survival than non-SR treatments, even for patients with multiple tumors [9, 10, 13, 22]. Furthermore, compared with TACE, SR significantly increases survival in select patients with BCLC stage B HCC [13, 23]. SR is a safe and effective therapy for select patients with resectable multiple or large $\mathrm{HCC}$ lesions in the same half-liver and sufficient liver reserve. Hence, SR may be considered for select patients who fit these criteria and could be recommended for patients with BCLC stage B disease.

Patients with BCLC stage $C$ disease have poor outcomes because of the presence of advanced HCC associated with major vascular invasion and/or extrahepatic metastasis. Sorafenib is the only recommended standard of care for advanced HCC based on the BCLC staging system. However, because of the large heterogeneity in the population with advanced-stage HCC, SR is no longer contraindicated and provides survival benefit [10, 22]. Moreover, several studies have demonstrated significantly favorable survival in HCC patients with major 

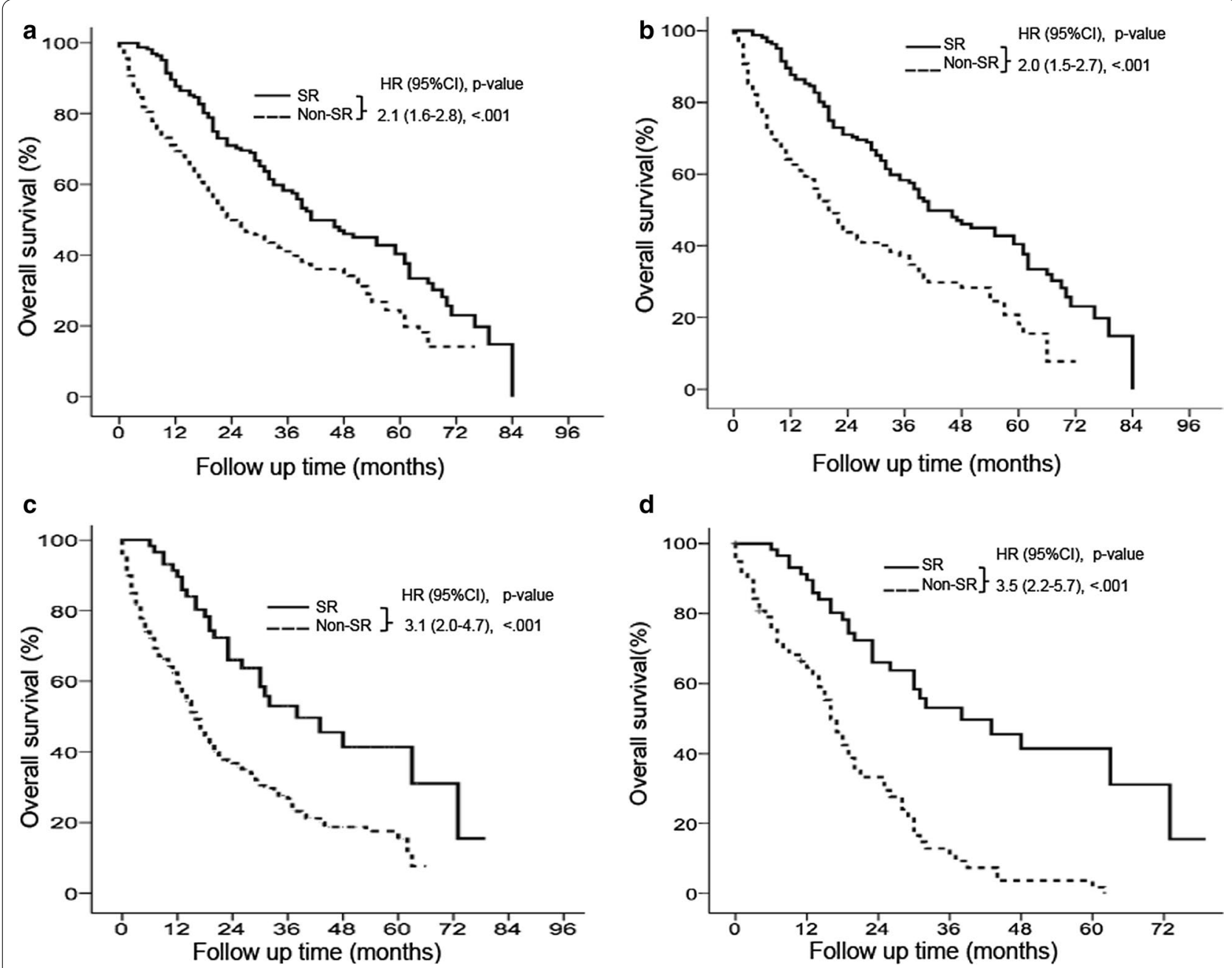

Fig. 4 Overall survival in patients with $B C L C$ stage 0 and A disease. Surgical resection (SR) resulted in significantly higher overall survival (OS) rates than non-SR treatments in BCLC stage $0(P<0.05)(\mathbf{a})$. After propensity score matching (PSM), SR still had significantly higher OS rates than non-SR treatments in BCLC stage $0(P<0.05)(\mathbf{b})$. SR resulted in significantly higher OS rates than non-SR treatments in BCLC stage $A(P<0.05)(\mathbf{c})$. After $P S M$, SR still had significantly higher OS rates than non-SR treatments in BCLC stage $\mathrm{A}(P<0.05)(\mathbf{d})$

vascular invasion, including the portal vein, hepatic vein and inferior vena cava, after SR [24-26]. Our results also confirmed the data from previous studies [10, 22, 24] and demonstrated that SR improved OS rates in patients with advanced-stage HCC. Therefore, meticulous and accurate selection criteria (HCC is located on the left or right lobe of liver, and portal vein tumor thrombosis in the segmental branch or first branch of portal vein can be excised in the same half-liver) should be established to identify individuals, among patients with vascular invasion, who would benefit most from SR. Hence, SR may also be considered for select patients with BCLC stage C HCC.

Liver function preservation, including $\mathrm{C}-\mathrm{P}$ class and cirrhosis, is an important non-oncological factor affecting OS. Poor liver function preservation decreases the efficacy of treatment and increases mortality. Our results showed that cirrhosis and $\mathrm{C}-\mathrm{P}$ class significantly affect $\mathrm{OS}$ in patients with $\mathrm{HCC}$ of various BCLC stages. Patients with cirrhosis easily develop portal hypertension, liver failure, and HCC. Additionally, patients with $\mathrm{C}-\mathrm{P}$ class $\mathrm{B}$ disease have low survival. It is important to treat liver disease using antiviral therapy and prevent liver disease progression.

Taiwan is a hyperendemic area for HBV-related liver diseases and HCC. HBV infection can result in hepatocarcinogenesis, and multiple mechanisms have been proposed, including the accumulation of genetic damage due to the induction of oxidative stress and immune-mediated hepatic inflammation. The integration of HBV DNA 
into the human genome occurs during the early steps of carcinogenesis and can induce alterations in cancerrelated gene expression and chromosomal instability [27, 28]. Our study demonstrated that $71.6 \%$ of HCC patients had HBV infection and that HBV infection significantly reduced OS rates in patients with BCLC stage $B$ and $C$ disease. In Taiwan, HBV-related HCC accounted for $88 \%$ of all cases before 1990, whereas from 1990 to 2000, the proportion of HBV-related HCC decreased to 66\% [29, 30]. Our study demonstrated that $71.6 \%$ of HCC patients had HBV infection, and the proportion of HBV-related HCC remained high in southern Taiwan. In addition, HBV infection significantly reduces OS rates in patients with BCLC stage B and C disease. Therefore, it is probable that HCC is caused not only by cirrhosis but also by HBV infection-induced hepatocarcinogenesis.

Our study has several limitations. First, as with all retrospective studies, there was some selection bias, including differences among patients regarding treatment decisions and the presence of incomplete data including alpha-fetoprotein, vascular invasion, extrahepatic metastases, performance status, and clinically relevant portal hypertension. Second, patients might receive multimodal treatments in a sequential manner, which would make direct comparison of every single treatment difficult in intermediate- and advanced-stage disease. Third, patients undergoing liver transplantation were not included because of the small sample size. Fourth, the concept of therapeutic hierarchy using the inverse probability of treatment weights and ITA.LI.CA staging will be further studied [31, 32].

\section{Conclusions}

Compared with nonsurgical treatments, SR significantly promoted survival benefits not only in very early- and early-stage but also in intermediate- and advanced-BCLC stage HCC. These results are valid in the cohorts with propensity score matching, and does not always represent results for all patients with intermediate- and advanced-BCLC stage HCC. More effort should be made to determine the proper selection criteria for SR in patients, especially in patients with intermediate- and advanced-stage disease. Additionally, the BCLC staging system should be further modified based on results from the clinic and responses to combinations of various treatment modalities.

\footnotetext{
Abbreviations

HCC: Hepatocellular carcinoma; BCLC: Barcelona Clinic Liver Cancer; AASLD: American Association for the Study of Liver Disease; ESAS: European Association for the Study of Liver; SR: Surgical resection; TACE: Transcatheter arterial chemoembolization; OS: Overall survival; RFA: Radiofrequency ablation; HAIC: Hepatic artery infusion chemotherapy; RT: Radiotherapy; BSC: Best supportive care; C-P class: Child-Pugh class; HBV: Hepatitis B virus; HCV: Hepatitis C virus;
}

PSM: Propensity score matching; Cl: Confidence interval; HR: Hazard ratio; ECOG: Eastern Cooperative Oncology Group.

\section{Supplementary Information}

The online version contains supplementary material available at https://doi. org/10.1186/s12876-021-01807-4.

Additional file 1: Figure S1. Overall survival in patients with BCLC stage $0, A, B$, and $C$ disease by Kaplan-Meier analysis. Surgical resection (SR) resulted in significantly higher overall survival than radiofrequency ablation (RFA) and transcatheter arterial chemoembolization (TACE) in BCLC stage $0(P<0.05)(A)$. SR resulted in significantly higher overall survival than RFA and TACE in BCLC stage $A(P<0.05)$ (B). SR resulted in significantly higher overall survival than RFA and TACE in BCLC stage $A(P<0.05)(B)$. SR resulted in significantly higher overall survival than RFA, TACE, and other treatment in $B C L C$ stage $B(P P<0.05)(C)$. SR resulted in significantly higher overall survival than RFA, TACE, target therapy, radiotherapy (RTO), hepatic artery infusion therapy (HAIC), and best support care (BSC) in BCLC stage $C(P<0.05)(D)$.

\section{Acknowledgements}

None.

\section{Authors' contributions}

YSC, PMH and HYL: study concept and design; acquisition of data; analysis and interpretation of data; drafting of the manuscript; $\mathrm{CMH}, \mathrm{GHL}, \mathrm{YCH}, \mathrm{ICL}, \mathrm{CYL}$, TCW, JHY, PH, YCL, YCW and KCH: study concept and design; critical revision of the manuscript for important intellectual content; administrative, technical, or material support; CWL: study concept and design; analysis and interpretation of data; critical revision of the manuscript for important intellectual content administrative, technical, or material support; study supervision. All authors approved the final version of the manuscript.

\section{Funding}

This study was supported by grants to Chih-Wen Lin from MOST (108-2314-B-214-006-MY2), the E-Da Hospital-National Taiwan University Hospital Joint Research Program (105-EDN13, 108-EDN11 and 109-EDN03), and the E-Da Hospital (EDAHP109044, EDAHP109045, EDAHP109053, EDPJ105056, EDPJ106094, EDPJ107076, EDPJ108069, and EDPJ109072). The funders had a role in study design, decision to publish and preparation of the manuscript. No additional external funding was received for this study.

Availability of data and materials

Data is available from the corresponding author upon reasonable request.

\section{Declarations}

\section{Ethics approval and consent to participate}

The study was conducted in accordance with the guidelines of the International Conference on Harmonization for Good Clinical Practice and was approved by the Ethics Committee of E-Da Hospital, I-Shou University (EMRP-107-130)

\section{Consent for publication}

Not applicable.

\section{Competing interests}

The authors declared that they have no competing interests.

\section{Author details}

${ }^{1}$ Division of Gastroenterology and Hepatology, E-Da Dachang Hospital, I-Shou University, No. 1, Yida Road, Jiaosu Village, Yanchao District, Kaohsiung 807, Taiwan, ROC. '2Division of Gastroenterology and Hepatology, Department of Medicine, E-Da Hospital, I-Shou University, Kaohsiung 824, Taiwan, ROC. ${ }^{3}$ Health Examination Center, E-Da Hospital, I-Shou University, Kaohsiung 824 Taiwan, ROC. ${ }^{4}$ School of Medicine, College of Medicine, I-Shou University, Kaohsiung 824, Taiwan, ROC. ${ }^{5}$ Department of Surgery, E-Da Hospital, I-Shou 
University, Kaohsiung 824, Taiwan, ROC. ${ }^{6}$ School of Chinese Medicine, College of Chinese Medicine, China Medical University, Taichung 404, Taiwan, ROC. ${ }^{7}$ Research Center for Traditional Chinese Medicine, China Medical University Hospital, Taichung 404, Taiwan, ROC. ${ }^{8}$ Department of Surgery, National Taiwan University Hospital, Taipei 100, Taiwan, ROC.

Received: 26 December 2020 Accepted: 6 May 2021 Published online: 14 May 2021

\section{References}

1. Yang JD, Roberts LR. Hepatocellular carcinoma: a global view. Nat Rev Gastroenterol Hepatol. 2010;7(8):448-58.

2. Omata M, Lesmana LA, Tateishi R, Chen PJ, Lin SM, Yoshida H, et al. Asian Pacific Association for the Study of the Liver consensus recommendations on hepatocellular carcinoma. Hepatol Int. 2010;4(2):439-74.

3. Edge SB, Compton CC, The American Joint Committee on Cancer. the 7th edition of the AJCC cancer staging manual and the future of TNM. Ann Surg Oncol. 2010;17(6):1471-4.

4. Llovet JM, Bru C, Bruix J. Prognosis of hepatocellular carcinoma: the BCLC staging classification. Semin Liver Dis. 1999;19(3):329-38.

5. Kudo M, Chung H, Osaki Y. Prognostic staging system for hepatocellular carcinoma (CLIP score): its value and limitations, and a proposal for a new staging system, the Japan Integrated Staging Score (JIS score). J Gastroenterol. 2003;38(3):207-15.

6. Bruix J, Sherman M. American Association for the Study of Liver D Management of hepatocellular carcinoma: an update. Hepatology. 2011;53(3):1020-2.

7. European Organisation For L, European Organisation For R. Treatment of C EASL-EORTC clinical practice guidelines: management of hepatocellular carcinoma. J Hepatol. 2012;56(4):908-43.

8. Ye SL, Chen RX. Comments on management of hepatocellular carcinoma: an update. Zhonghua Gan Zang Bing Za Zhi. 2011;19(4):251-3.

9. Forner A, Gilabert M, Bruix J, Raoul JL. Treatment of intermediate-stage hepatocellular carcinoma. Nat Rev Clin Oncol. 2014;11(9):525-35.

10. Ho MC, Hasegawa K, Chen XP, Nagano H, Lee YJ, Chau GY, et al. Surgery for intermediate and advanced hepatocellular carcinoma: a consensus report from the 5th Asia-Pacific primary liver cancer expert meeting (APPLE 2014). Liver Cancer. 2016;5(4):245-56.

11. Koh YX, Tan HL, Lye WK, Kam JH, Chiow AKH, Tan SS, et al. Systematic review of the outcomes of surgical resection for intermediate and advanced Barcelona Clinic Liver Cancer stage hepatocellular carcinoma: a critical appraisal of the evidence. World J Hepatol. 2018;10(6):433-47.

12. Chang YJ, Chung KP, Chang YJ, Chen LJ. Long-term survival of patients undergoing liver resection for very large hepatocellular carcinomas. Br J Surg. 2016;103(11):1513-20.

13. Yin L, Li H, Li AJ, Lau WY, Pan ZY, Lai EC, et al. Partial hepatectomy vs. transcatheter arterial chemoembolization for resectable multiple hepatocellular carcinoma beyond Milan Criteria: a RCT. J Hepatol. 2014;61(1):82-8.

14. Lee HS, Kim KM, Yoon JH, Lee TR, Suh KS, Lee KU, et al. Therapeutic efficacy of transcatheter arterial chemoembolization as compared with hepatic resection in hepatocellular carcinoma patients with compensated liver function in a hepatitis B virus-endemic area: a prospective cohort study. J Clin Oncol. 2002;20(22):4459-65.

15. Lin CW, Chen YS, Lin CC, Lee PH, Lo GH, Hsu CC, et al. Significant predictors of overall survival in patients with hepatocellular carcinoma after surgical resection. PLOS ONE. 2018.

16. Fujii H, Itoh Y, Ohnishi N, Sakamoto M, Ohkawara T, Sawa Y, et al. Factors associated with the overall survival of elderly patients with hepatocellular carcinoma. World J Gastroenterol. 2012;18(16):1926-32.
17. Pugh RN, Murray-Lyon IM, Dawson JL, Pietroni MC, Williams R. Transection of the oesophagus for bleeding oesophageal varices. Br J Surg. 1973:60(8):646-9.

18. Yin Z, Jin H, Ma T, Zhou Y, Yu M, Jian Z. A meta-analysis of long-term survival outcomes between surgical resection and radiofrequency ablation in patients with single hepatocellular carcinoma $=2 \mathrm{~cm}$ (BCLC very early stage). Int J Surg. 2018;56:61-7.

19. Sarin SK, Kumar M, Lau GK, Abbas Z, Chan HL, Chen CJ, et al. Asian-Pacific clinical practice guidelines on the management of hepatitis B: a 2015 update. Hepatol Int. 2016;10(1):1-98.

20. Pompili M, Saviano A, de Matthaeis N, Cucchetti A, Ardito F, Federico B, et al. Long-term effectiveness of resection and radiofrequency ablation for single hepatocellular carcinoma $</=3 \mathrm{~cm}$. Results of a multicenter Italian survey $=3 \mathrm{~cm}$. Results of a multicenter Italian survey. J Hepatol. 2013;59(1):89-97

21. Bolondi L, Burroughs A, Dufour JF, Galle PR, Mazzaferro V, Piscaglia F, et al. Heterogeneity of patients with intermediate (BCLC B) Hepatocellular Carcinoma: proposal for a subclassification to facilitate treatment decisions. Semin Liver Dis. 2012;32(4):348-59.

22. Guo H, Wu T, Lu Q, Li M, Guo JY, Shen Y, et al. Surgical resection improves long-term survival of patients with hepatocellular carcinoma across different Barcelona Clinic Liver Cancer stages. Cancer Manag Res. 2018;10:361-9.

23. Qi X, Wang D, Su C, Li H, Guo X. Hepatic resection versus transarterial chemoembolization for the initial treatment of hepatocellular carcinoma: a systematic review and meta-analysis. Oncotarget. 2015;6(21):18715-33.

24. Zhang XP, Wang K, Li N, Zhong CQ, Wei XB, Cheng YQ, et al. Survival benefit of hepatic resection versus transarterial chemoembolization for hepatocellular carcinoma with portal vein tumor thrombus: a systematic review and meta-analysis. BMC Cancer. 2017;17(1):902.

25. Kudo M, Izumi N, Ichida T, Ku Y, Kokudo N, Sakamoto M, et al. Report of the 19th follow-up survey of primary liver cancer in Japan. Hepatol Res. 2016;46(5):372-90.

26. Wang Y, Yuan L, Ge RL, Sun Y, Wei G. Survival benefit of surgical treatment for hepatocellular carcinoma with inferior vena cava/right atrium tumor thrombus: results of a retrospective cohort study. Ann Surg Oncol. 2013;20(3):914-22.

27. Cougot D, Neuveut C. Buendia MA HBV induced carcinogenesis. J Clin Virol. 2005;34(Suppl 1):75-8

28. Tarocchi M, Polvani S, Marroncini G, Galli A. Molecular mechanism of hepatitis B virus-induced hepatocarcinogenesis. World J Gastroenterol. 2014;20(33):11630-40.

29. Chen DS. Hepatocellular carcinoma in Taiwan. Hepatol Res. 2007;37(Suppl 2):101-5.

30. Lu SN, Su WW, Yang SS, Chang TT, Cheng KS, Wu JC, et al. Secular trends and geographic variations of hepatitis $B$ virus and hepatitis $C$ virus-associated hepatocellular carcinoma in Taiwan. Int J Cancer. 2006;119(8):1946-52

31. Vitale A, Trevisani F, Farinati F, Cillo U. Treatment of hepatocellular carcinoma in the precision medicine era: from treatment stage migration to therapeutic hierarchy. Hepatology. 2020;72(6):2206-18.

32. Vitale A, Farinati F, Pawlik TM, Frigo AC, Giannini EG, Napoli L, et al. The concept of therapeutic hierarchy for patients with hepatocellular carcinoma: a multicenter cohort study. Liver Int. 2019;39(8):1478-89.

\section{Publisher's Note}

Springer Nature remains neutral with regard to jurisdictional claims in published maps and institutional affiliations. 